\title{
Testes utilizados para avaliação respiratória nas doenças neuromusculares
}

\section{Tests used to evaluate respiratory function in neuromuscular diseases}

\author{
Natasha Nicholson de Santa Maria ${ }^{1}$, Erica Marques Zanelli', Marina Brito Silva ${ }^{1}$, Sabrina Kyoko de \\ Paula Asa ${ }^{1}$, Francis Meire Fávero², Marcia Maiumi Fukujima³, Acary Souza Bulle Oliveira ${ }^{4}$, Sissy \\ Veloso Fontes ${ }^{5}$.
}

\section{RESUMO}

O objetivo deste estudo consiste em identificar e caracterizar os principais testes utilizados na avaliação da função pulmonar de pacientes com doenças neuromusculares citados em artigos científicos publicados nos últimos 40 anos. Utilizou-se de revisão bibliográfica incluindo estudos publicados nos anos de 1965 a 2005 encontrados nas bases de dados MEDLINE, PUBMED, EMBASE, LILACS e SCIELO que utilizaram descritores na língua portuguesa (doenças neuromusculares, espirometria, capacidade vital, valores de referência) e na inglesa (neuromuscular disease, spirometry, vital capacity reference values). Foram encontrados 9 testes subdivididos em medidas, valores de referência e utilidade. Os testes citados foram: testes de função pulmonar que incluem medidas espirométricas, utilizados para avaliar volumes pulmonares, presença de obstrução de vias aéreas, sinais de fraqueza e fadiga dos músculos respiratórios; testes que avaliam a força dos músculos respiratórios, utilizados para avaliar a força dos músculos respiratórios e a atividade diafragmática isolada; teste que avalia a atividade elétrica de nervos, junção neuromuscular e músculos relacionados a respiração; testes que avaliam o limiar de fadiga dos músculos respiratórios; teste que avalia o grau de relaxamento dos músculos respiratórios, utilizado para analisar a recuperação muscular; testes que avaliam a eficácia da tosse; análise da concentração de gases arteriais, capnografia e oximetria de pulso, utilizadas para avaliar principalmente retenção de $\mathrm{CO}_{2}$ e presença de hipoxemia durante o sono, respectivamente.

\section{Unitermos: Doenças neuromusculares, Fisioterapia, Espirometria, Capacidade vital, Valores de referência.}

Citação: Maria NNS, Zanelli EM, Silva MB, Asa SKP, Fávero FM, Fukujima MM, Oliveira ASB, Fontes SV. Testes utilizados para avaliação respiratória nas doenças neuromusculares. Rev Neurocienc 2007; 15(1):60-69.

\section{SUMMARY}

The aim of the present study was to identify and analyze the main tests used in the pulmonary function assessment of patients with neuromuscular diseases presented in published articles in the last 40 years. We searched the published articles from 1965 to 2005, in the databases MEDLINE, PUBMED, EMBASES, LILACS, and SCIELO that used keywords in Portuguese and in English (neuromuscular disease, spirometry, vital capacity reference values). We found 9 tests related to pulmonaty function, subdivided in measures, values of reference, and utility. The cited tests were: tests for pulmonary function that includes measured spirometric, they were used to assess pulmonary volumes, presence of airway obstruction, signals of weakness of the respiratory muscles and endurance; tests for respiratory muscles strength, they were used to assess the force of inspiratory

Trabalho realizado no Setor de Investigação de Doenças Neuromusculares da Disciplina de Neurologia Clínica da Universidade Federal de São Paulo (UNIFESP)

1. Fisioterapeuta do Hospital Israelita Albert Einstein, Especialista em Intervenção Fisioterapêutica nas Doenças Neuromusculares pela UNIFESP.

2. Fisioterapeuta, Mestre em Neurociências pela UNIFESP, Coordenadora do Curso de Especialização em Intervenção Fisioterapêutica nas Doenças Neuromusculares da UNIFESP.

3. Neurologista da Disciplina de Medicina de Urgência e Medicina Baseada em Evidências da UNIFESP, Doutora em Ciências pela UNIFESP.

4. Neurologista, Professor Filiado da Disciplina de Neurologia da UNIFESP.

5 Fisioterapeuta da Disciplina de Neurologia da UNIFESP, Educadora Física e Doutora em Ciências pela UNIFESP.

Endereço para correspondência: Sissy Veloso Fontes, R: Francisco Tapajós, 513 apto. 122, Vila Santo Estéfano - CEP: 04153001 -São Paulo, SP. E-mail: sissyfontes@gmail.com

Trabalho recebido em 26/04/2006 • Revisão: de 27/04/2006 a 18/07/2006 • Aprovado em 19/07/2006 • Conflito de interesses: não 
and expiratory muscles or diaphragmatic activity; test for electric activity of nerves, neuromuscular junction, and muscles; tests for respiratory muscles threshold fatigue; tests for relaxation degree of respiratory muscles, they were used to assess muscular recovery; tests for effectiveness of the cough; analysis of the arterial gases concentration, capnografy, and pulse oximetry they were used to assess Co2 retention and presence of hypoxemia during sleep, respectively.

Keywords: Neuromuscular diseases, Physical therapy, Spirometry, Vital capacity, Reference values.

Citation: Maria NNS, Zanelli EM, Silva MB, Asa SKP, Fávero FM, Fukujima MM, Oliveira ASB, Fontes SV. Tests used to evaluate respiratory function in neuromusculares diseases. Rev Neurocienc 2007; 15(1):60-69.

\section{INTRODUÇÃO}

As doenças neuromusculares (DNM) compreendem o acometimento de estruturas como: neurônio motor inferior, nervo periférico, junção mioneural e músculo estriado esquelético. Sendo assim, as manifestações clínicas variam e, são dependentes da estrutura neuroanatômica acometida ${ }^{1}$.

A fraqueza dos músculos respiratórios é freqüente nas doenças neuromusculares (DNM) e está associada diretamente a morbidade e mortalidade destes pacientes $^{2-4}$. As DNM mais comuns que causam fraqueza dos músculos respiratórios e/ou falência respiratória são: miastenia grave, síndrome de Guillan Barré, poliomielite, distrofia muscular de Duchenne e esclerose lateral amiotrófica².

O comprometimento dos grupos musculares respiratórios traz, como conseqüências a hipoventilação associada à retenção de muco brônquico, podendo evoluir para atelectasia e predisposição a infecções respiratórias de repetição. Estas complicações respiratórias interferem na condição clínica geral destes pacientes e, podem levar à fadiga e piora da fraqueza muscular generalizada 5 .

O grau de disfunção muscular respiratória varia entre as diversas DNM e podem causar sintomas que vão da dispnéia aos esforços até o desconforto respiratório grave. Podemos encontrar pacientes com DNM crônica sem sintomas respiratórios, porém com fraqueza muscular respiratória evidente6. Além disso, o comprometimento respiratório também varia de acordo com a postura adotada como, por exemplo, sentada, decúbito dorsal ou decúbito lateral ${ }^{7}$.

A avaliação freqüente da função pulmonar destes pacientes é importante para eleger as melhores opções terapêuticas, de maneira a evitar a falência respiratória precoce e, maximizar a sobrevida destes pacientes ${ }^{8,9}$.

O conjunto de sinais e sintomas clínicos relacionados à função respiratória é freqüentemente e, primariamente investigado durante a anamnese e exame físico, antes da solicitação de exames e ou testes subsidiários.

A maioria dos pacientes com DNM apresenta fraqueza dos músculos estriados esqueléticos apendiculares antes dos músculos respiratórios sendo, em poucos casos, o comprometimento respiratório, a queixa ou sintoma primário9.

A dispnéia nas DNM evolui de maneira lenta e insidiosa caracterizada, inicialmente pela hipoventilação noturna, principalmente durante o sono REM e na posição supina. Portanto, é de fundamental importância que o examinador atente para os sinais de hipoventilação noturna: distúrbio do sono com despertar freqüente ou insônia, roncos, asfixia e ou sensação de sufocar mesmo na ausência de queixa de dispnéia. A ortopnéia, dispnéia ou piora do padrão respiratório em posição supina é uma queixa freqüente de pacientes com extrema fraqueza do diafragma. Neste caso, o paciente refere que só consegue dormir com mais de um travesseiro sob a cabeça. Com um sono ineficaz, os pacientes queixam-se de hipersonolência diurna, indisposição, depressão, dor de cabeça pela manhã, perda de memória, sensação de cansaço, fadiga e apresentam uma aparência extremamente exausta ${ }^{10}$.

Estes relatos podem estar acompanhados por redução do apetite e rápida perda de peso. Pode ocorrer distúrbio de deglutição associado à disfunção pulmonar. Pacientes e cuidadores notam tosse e engasgos freqüentes durante a deglutição, o que indica aspiração ou microaspiração ${ }^{11}$.

A fraqueza dos músculos respiratórios primários aumenta o uso da musculatura respiratória coadjuvante como, os esternocleidomastóideos e escalenos. O uso desta musculatura indica que a carga sobre os músculos respiratórios é maior que sua capacidade de contração $0^{9,10}$. Se a fraqueza muscular respiratória progredir, segue-se o aparecimento de respiração paradoxal, a mais típica alteração do exame físico, sendo este um sinal de fraqueza diafragmática importante ${ }^{11}$.

A perda da força muscular progressiva pode levar à alteração da caixa torácica e coluna vertebral. A escoliose, hipercifose, cifoescoliose e retração esternal2,6 são alterações freqüentes que, em associação com a perda de força muscular respiratória causa diminuição da expansibilidade da caixa torácica e aumento do trabalho respiratório ${ }^{2,5,6,11}$.

A anamnese e exame físico são pouco sensíveis e específicos para quantificar o comprometimento dos músculos respiratórios, porque alterações do exame físico só aparecem quando a força muscular é menor 
do que $25 \%$ do normal4. Pacientes com com esclerose lateral amiotrófica (ELA) com capacidade vital forçada menor que $30 \%$ do predito, podem não apresentar sintomas de dispnéia ${ }^{12}$. Portanto, os sintomas de dispnéia não são suficientes para identificar e caracterizar as disfunções pulmonares nestes casos, o que torna necessário a utilização de exames subsidiários nos casos de $\mathrm{DNM}^{12}$.

Dentre os exames subsidiários destacam-se os exames de imagem, como o raio-x de tórax e o ultra-som de tórax. Estes exames corroboram em relação à dinâmica ventilatória, mas não são sensíveis o suficiente para predizer o grau de disfunção muscular respiratória ${ }^{13}$.

Além destes exames, existem diversos testes utilizados para avaliação da função pulmonar de pacientes com DNM. Estes objetivam identificar e ou quantificar a gravidade da fraqueza dos músculos respiratórios, o comprometimento da troca gasosa, a capacidade de manter o claerence pulmonar; permitem estabelecer a causa de dispnéia e a presença de fadiga; determinam o momento adequado para inserir a ventilação mecânica pulmonar não invasiva e, fornecem informação sobre a função pulmonar do paciente para suportar, se necessário um procedimento cirúrgico.

O conhecimento e aplicação destes testes na prática clínica são de fundamental importância para fornecer a assistência adequada. Sendo assim, o objetivo deste estudo consiste em identificar e caracterizar os principais testes utilizados na avaliação da função pulmonar de pacientes com doenças neuromusculares citados em artigos científicos publicados nos últimos 40 anos.

\section{MÉTODO}

Para identificação e caracterização dos testes utilizados na avaliação da respiração de pacientes com doenças neuromusculares, utilizou-se de revisão bibliográfica incluindo artigos científicos publicados nos anos de 1965 a 2005 encontrados nas bases de dados MEDLINE, PUBMED, EMBASE, SCIELO e LILACS que utilizaram descritores na língua portuguesa (doenças neuromusculares, espirometria, capacidade vital, valores de referência) e na inglesa (neuromuscular disease, spirometry, vital capacity, reference values) e por busca manual das referências citadas nos artigos encontrados. A estratégia de busca utilizada tanto na MEDLINE quanto na SCIELO foi: descritor de assunto="doenças neuromusculares" AND Aspectos = Uso diagnóstico (/DU) AND Espécie = Humanos. Na PUBMED foi: neuromuscular disease, meansurement AND limits = humans. E na EMBASE foi: "neuromuscular disease" lexp OR 'neuromuscular disease' AND measurement AND [humans] / lim 1965 a 2005.

Foram excluídos os estudos que utilizaram instrumentos de avaliação da função motora, qualidade de vida e, experimentos com animais.

Após a seleção dos artigos que preenchiam os critérios de inclusão, foram extraídas informações referentes aos testes de função pulmonar utilizados e descritos e estes foram tabulados segundo o tipo de teste, medidas, valores de referência e utilidade.

\section{RESULTADOS}

Foram encontrados 56 artigos científicos que citavam um ou vários tipos de testes para avaliação da função pulmonar de pacientes com DNM. Destes foram excluídos 38 devido não incluírem adequadamente a descrição e ou a utilidade dos testes citados, ou devido os testes terem sido utilizados para confirmar indicação de ventilação mecânica e ou para indicar gastrostomia. Os 18 artigos incluídos reportaram no total 9 testes de avaliação da função pulmonar. Os dados relativos a cada teste foram extraídos e expressos segundo as medidas, valores de referência e utilidade (Tabela 1).

\section{DISCUSSÃO}

A falta de especificidade e sensibilidade da anamnese, do exame físico e dos testes subsidiários mostra a necessidade de testes que identificam e quantificam o grau de disfunção ventilatória de pacientes com DNM.

Os diferentes testes encontrados na literatura podem ser agrupados em testes de: função pulmonar (espirometria); força dos músculos respiratórios e atividade diafragmática isolada; atividade elétrica de nervos, junção neuromuscular e músculos; fadiga dos músculos respiratórios; eficácia da tosse; e gases arteriais, principalmente durante o sono.

Os testes citados na literatura, como os mais eficazes em predizer a fraqueza dos músculos respiratórios, são as medidas invasivas (pressões esofágica, gástrica e transdiafragmática), pois traduzem, exclusivamente o gradiente de pressão dos músculos respiratórios. Os menos invasivos, como a espirometria e a avaliação indireta da força muscular respiratória (pressões inspiratórias e expiratórias máximas) refletem todas as forças envolvidas na mecânica ventilatória.

As medidas durante uma manobra nasal podem ser mais fidedignas em se tratando de pacientes com DNM, principalmente na presença de comprometimento bulbar, pois a fraqueza dos músculos orofaríngeos prejudicam o resultado de alguns testes.

\section{Testes de Função Pulmonar (espirometria)}

Os testes de função pulmonar consistem em medidas da quantidade de ar inspirado e expirado expressa em litros, que avaliam a capacidade pulmonar em mover 
Tabela 1. Tabulação dos testes utilizados para avaliação da função pulmonar em pacientes com DNM segundo o tipo, medidas, valores de referência e utilidade.

\begin{tabular}{|c|c|c|c|c|}
\hline \multirow{2}{*}{ Tipo de teste } & \multirow{2}{*}{ Medidas } & \multicolumn{2}{|c|}{ Valores de Referência (Adulto) } & \multirow{2}{*}{ Utilidade } \\
\hline & & $\begin{array}{ll}\mathbf{H} \\
\end{array}$ & $\mathbf{M}$ & \\
\hline \multirow[t]{6}{*}{ Espirometria } & CVF & $\begin{array}{c}\text { Estatura x 0,059-idade } \mathrm{x} \\
0,0229-4,569\end{array}$ & $\begin{array}{l}\text { Estatura } \times 0,0433 \text { - idade } x \\
0,0164-2,967\end{array}$ & Avaliar volumes pulmonares ${ }^{18}$ \\
\hline & $\mathrm{VEF}_{1}$ & \begin{tabular}{|c|} 
Estatura $\times 0,0473-$ idade $x$ \\
$0,0281-3,145$
\end{tabular} & $\begin{array}{l}\text { Estatura } \times 0,0338-\text { idade } x \\
\quad 0,0210-1,782\end{array}$ & Avaliar obstrução pulmonar ${ }^{18}$ \\
\hline & $\mathrm{VEF}_{1} / \mathrm{CVF}$ & - $\log$ idade $\times 0,1198+4,854$ & $4,8707-\log$ idade $\times 0,1212$ & $\begin{array}{c}\text { Determinar o comprometimento pul- } \\
\text { monar }{ }^{18}\end{array}$ \\
\hline & FEF25-75\% & $\begin{array}{l}\text { log estatura } \times 2,0020-\log \\
\text { idade } \times 0,6977-0,3279\end{array}$ & $\begin{array}{l}\text { log estatura } \times 1,2843-\log \\
\text { idade } \times 0,6546-0,30208\end{array}$ & Obstrução de VAS mais proximais' ${ }^{18}$ \\
\hline & FEF75-85\% & $\begin{array}{l}\text { log estatura } \times 2,2537-\log \\
\text { idade } \times 1,164-7,2889\end{array}$ & $\begin{array}{l}\log \text { estatura } \times 2,0786-\log \\
\text { idade } \times 1,2686-6,1431\end{array}$ & Obstrução de VAS mais distais ${ }^{18}$ \\
\hline & Curvas fluxo-volume & ---- & ---- & $\begin{array}{l}\text { Fraqueza dos músculos respiratóri- } \\
\qquad \mathrm{OS}^{15,26,27}\end{array}$ \\
\hline \multirow{10}{*}{$\begin{array}{c}\text { Força muscular res- } \\
\text { piratória }\end{array}$} & \multirow{2}{*}{$\begin{aligned} & \text { VVM } \\
& \text { Manobra Oral: } \text { PImáx } \\
& \text { PEmáx } \\
&\end{aligned}$} & $>10 \mathrm{~L} / \mathrm{min}$ & $>10 \mathrm{~L} / \mathrm{min}$ & Teste de esforço ${ }^{16}$ \\
\hline & & $-60 \mathrm{cmH}_{2} \mathrm{O}$ & $-60 \mathrm{CmH}_{2} \mathrm{O}$ & Avalia força muscular inspiratória ${ }^{15,32}$ \\
\hline & \multirow{3}{*}{$\begin{array}{r}\text { Manobra nasal: Pnas,sn } \\
\text { Pes,sn } \\
\text { Pdi,sn }\end{array}$} & $90 \mathrm{cmH}_{2} \mathrm{O}$ & $90 \mathrm{cmH}_{2} \mathrm{O}$ & Avalia força muscular expiratória ${ }^{15}$ \\
\hline & & $-70 \mathrm{cmH}_{2} \mathrm{O}$ & $-60 \mathrm{CmH}_{2} \mathrm{O}$ & Avalia força muscular inspiratória ${ }^{15}$ \\
\hline & & $-80 \mathrm{cmH}_{2} \mathrm{O}$ & $-70 \mathrm{cmH}_{2} \mathrm{O}$ & Avalia a força muscular inspiratória ${ }^{13}$ \\
\hline & & $100 \mathrm{cmH}_{2} \mathrm{O}$ & $80 \mathrm{cmH}_{2} \mathrm{O}$ & Avalia força muscular diafragmática ${ }^{15}$ \\
\hline & Pdi & $10 \mathrm{cmH}_{2} \mathrm{O}$ & $10 \mathrm{cmH}_{2} \mathrm{O}$ & Gold standart - força diafragmática ${ }^{30,34}$ \\
\hline & $\Delta \mathrm{Pga} / \Delta \mathrm{Pdi}$ & + & + & $\begin{array}{l}\text { Respiração paradoxal/atividade di- } \\
\text { afragmática }\end{array}$ \\
\hline & $\begin{array}{l}\text { Pdi durante EEM do nervo } \\
\text { frênico }\end{array}$ & $8.8-33 \mathrm{cmH}_{2} \mathrm{O}$ & $8.8-33 \mathrm{cmH}_{2} \mathrm{O}$ & Atividade muscular diafragmática ${ }^{13,15}$ \\
\hline & $\begin{array}{c}\text { Pdi durante EM do nervo } \\
\text { frênico }\end{array}$ & $20 \mathrm{cmH}_{2} \mathrm{O}$ & $20 \mathrm{cmH}_{2} \mathrm{O}$ & $\begin{array}{l}\text { Avalia atividade muscular respira- } \\
\text { tória }^{15,36}\end{array}$ \\
\hline \multirow{3}{*}{$\begin{array}{c}\text { Atividade neuromus- } \\
\text { cular } \\
\text { Fadiga muscular } \\
\text { respiratória } \\
\end{array}$} & $\begin{array}{l}\text { EMG dos músculos respi- } \\
\text { ratórios }\end{array}$ & & --- & Nervos, JNM intrínseca dos músculos ${ }^{37}$ \\
\hline & TTdi & $>0.15$ & $>0.15$ & Limiar de fadiga muscular respiratória ${ }^{38}$ \\
\hline & Pdi/Pdimáx & $\geq 0.4$ & $\geq 0.4$ & Limiar de fadiga muscular respiratória ${ }^{38}$ \\
\hline \multirow{3}{*}{$\begin{array}{l}\text { Relaxamento do } \\
\text { diafragma } \\
\text { Eficácia da tosse }\end{array}$} & Diminuição da Pdi & -------- & -------- & Analisa a recuperação muscular ${ }^{39}$ \\
\hline & PFE & $>160 \mathrm{I} / \mathrm{min}$ & $>160 \mathrm{I} / \mathrm{min}$ & Avaliar a eficácia da tosse ${ }^{40}$ \\
\hline & PFT & $>100 \mathrm{I} / \mathrm{min}$ & $>100 \mathrm{l} / \mathrm{min}$ & Avaliar a eficácia da tosse ${ }^{40}$ \\
\hline \multirow{4}{*}{ Gasometria arterial } & Pga durante tosse & $>160 \mathrm{cmH}_{2} \mathrm{O}$ & $>120 \mathrm{cmH}_{2} \mathrm{O}$ & $\begin{array}{c}\text { Medida não volátil da eficácia da } \\
\text { tosse }{ }^{15,41}\end{array}$ \\
\hline & $\mathrm{PaCO2}$ & $<45 \mathrm{mmHg}$ & $<45 \mathrm{mmHg}$ & Avaliar concentração de C02 arterial43 \\
\hline & $\mathrm{PaO2}$ & $>80 \mathrm{mmHg}$ & $>80 \mathrm{mmHg}$ & Avaliar concentração de 02 arterial| ${ }^{42,43}$ \\
\hline & Sat02 & $>90 \%$ & $>90 \%$ & $\begin{array}{c}\text { Avaliar saturação de } 02 \text { da hemoglo- } \\
\text { bina }{ }^{42,43}\end{array}$ \\
\hline Capnografia & PetC02-PaC02 & $<10 \mathrm{mmHg}$ & $<10 \mathrm{mmHg}$ & $\begin{array}{c}\text { Avaliar retenção de CO2 durante } 0 \\
\text { sono } 04,46\end{array}$ \\
\hline Oximetria de pulso & Sap02 & $<80 \%$ & $<80 \%$ & Avaliar hipoxemia durante 0 sono $0^{44,46}$ \\
\hline
\end{tabular}

CVF- capacidade vital forçada; VEF1- volume expiratório forçado no primeiro segundo; FEF $25-75 \%, 75-85 \%, 50 \%$ fluxo expiratório forçado em 25 à $75 \%, 75$ à $85 \%$ e $50 \%$ da curva de fluxo expiratório; TEF $25-75 \%$ tempo expiratório em 25 à $75 \%$ da curva de fluxo; VVM - ventilação voluntária máxima; PImax e PEmax pressão inspiratória e expiratória máximas, Pnmax - pressão nasal máxima; Pneso - pressão nasal esofágica; Pndi - pressão transdiafragmática durante uma manobra nasal; Pdi - pressão transdiafragmática; $\triangle P$ ga / $\triangle P$ di - índice de Gilbert; EEM do nervo frênico - estimulação elétrica magnética do nervo frênico; $E M$ do nervo frênico - estimulação magnética do nervo frênico; EMG dos músculos respiratórios - eletromiografia dos músculos respiratórios; TTdi - Índice Tensão-Tempo; Pdi/Pdimáx - pressão transdiafragmatica para realizar um dado volume corrente (Pdi) sobre a pressão transdiafragmática em uma inspiração máxima (Pdimáx); PFE - pico de fluxo expiratório; PFT - pico de fluxo de tosse; PaCO2 - pressão parcial arterial de oxigênio; $\mathrm{PaO} 2$ - pressão parcial arterial de dióxido de carbono; SatO2 - saturação arterial de oxigênio; PetCO2-PaCO2 - diferença entre PaCO2 e PetCO2; SapO2 - saturação de pulso de oxigênio, $L=$ litros, $L / S=$ litros por segundo. 
rapidamente grandes quantidades de ar e presença de obstrução de vias aéreas ${ }^{14}$. Estes incluem as seguintes medidas: capacidade vital forçada (CVF), volume expiratório forçado no primeiro segundo $\left(\mathrm{VEF}_{1}\right)$, fluxo expiratório forçado em 25 a $75 \%$ e, 75 a 85\% da curva de fluxo expiratório (FEF25-75\%, FEF75-85\%) ${ }^{15}$.

A alteração da função pulmonar nas DNM é causada basicamente por redução da capacidade de expandir a caixa torácica, gerando menor pressão negativa necessária para produzir fluxo inspiratório e, conseqüente redução das propriedades viscoelásticas do pulmão ${ }^{10,11}$. Este mecanismo caracteriza um padrão restritivo nos testes de função pulmonar ${ }^{5,6,9}$. Sendo assim, o desvio da curva de pressão-volume para a direita e para baixo em altos volumes pulmonares indica redução da complacência pulmonar ${ }^{16}$

Os valores obtidos com a espirometria são interpretados através da comparação com valores estudados em amostra selecionada de uma determinada população de referência ${ }^{17}$. Estes valores são baseados em modelos de regressão linear que permitem a formulação de equações para idade, sexo, peso, altura ${ }^{16-22}$. Muitas equações de referência foram propostas para espirometria, mas como existem variações entre os valores de normalidade de cada amostra, estas equações podem subestimar os valores espirométricos analisados, especialmente nos extremos e nos intervalos entre as idades. A escolha dos valores de referência é essencial no diagnóstico e tratamento das $\mathrm{DNM}^{22} \mathrm{e}$, a conseqüência de uma escolha errada é um aumento na variabilidade dos valores previstos ${ }^{17}$

Muitas tabelas de valores de referência, assim como, as equações lineares são descritas na literatura ${ }^{16-22}$. No Brasil, valores previstos para os diversos parâmetros da curva expiratória forçada. foram estipulados em estudo realizado por Pereira e cols. ${ }^{18}$, sendo os valores obtidos, semelhantes aos do estudo realizado nos EUA por Dockery e cols. ${ }^{23}$.

Na população pediátrica, as equações dos valores preditos apresentam uma grande variação entre as idades. Estas equações utilizam idade e altura como variáveis independentes para os valores de $V_{E F}$ e CVF e, estes são transformados em logaritmos, afim de tornar linear as variações entre idade e altura ${ }^{24}$. No Brasil não foram encontrados estudos com valores de referência para a população pediátrica. O estudo europeu de Quanjer e cols. apresenta o melhor predito, além de sugerir a associação logarítmica entre a maturação pulmonar e a altura da criança como um modelo apropriado ${ }^{24}$.

Pacientes menores do que 6 anos podem apresentar valores mal interpretados, e diversos artefatos podem ser encontrados. Durante a adolescência, há uma relação linear entre o crescimento pulmonar e o crescimento somático, devendo ser considerado a relação entre idade, peso e altura. No entanto, esta variação pode não ser encontrada nos pacientes com DNM, especialmente na distrofia muscular de Duchenne 22 .

Para a realização das medidas, os indivíduos devem permanecer sentados confortavelmente com um clipe nasal e, instruídos sobre o procedimento. Os testes devem ser realizados com espirômetro calibrado diariamente. Pelo menos três manobras de expiração forçada são utilizadas para a análise dos dados, considerandose duas curvas que não diferem nas medidas de VEF e CVF por mais de $5 \%$ ou $100 \mathrm{ml}$. Os maiores valores adquiridos devem ser considerados para a análise dos resultados ${ }^{15,18}$. Devem ser excluídas curvas com artefatos de esforço submáximo na moforlogia, tais como vazamento de ar ou tosse ${ }_{22}$

A CVF esta relacionada com a sobrevida de pacientes com DNM ${ }^{20}$. A velocidade de redução da CVF varia entre estes pacientes, mas tende a ser linear. A velocidade é descrita entre 2.5 a $8.3 \%$ dos valores preditos por mês, com uma média de $3.5 \%$.

É importante lembrar que no início do comprometimento respiratório nas DNM, a função pulmonar é normal ou relativamente preservada por um período de tempo. Com o progredir da doença, o comprometimento pulmonar pode evoluir rapidamente com uma brusca redução da CVF em apenas 6 meses. A CVF < 50\% do predito é um importante estágio da falência respiratória, onde é de vital importância a introdução do suporte ventilatório ${ }^{15,16}$

Na maioria dos indivíduos normais, a CVF em posição supina é de 5 a $10 \%$ menor do que em ortostatismo ${ }^{15}$, porém, durante a fraqueza dos músculos respiratórios, esta redução é mais intensa. Em pacientes com paralisia diafragmática bilateral, a capacidade vital pode cair $50 \%$ do predito quando em posição supina. A redução da CVF de $25 \%$ ou mais entre a posição sentada ou supina tem sido um indicador sensível de fraqueza diafragmática6. Esta mudança possui uma especificidade e sensibilidade de $90 \%$ e $79 \%$ respectivamente, para o diagnóstico de fraqueza diafragmática ${ }^{5}$.

Em indivíduos saudáveis a comparação entre idade e VEF ${ }_{1}$ expressa uma perda anual esperada. De forma linear, o VEF1 cai $28 \mathrm{ml} /$ ano após os 25 anos de idade ${ }^{18}$. Nas DNM a redução da $V_{E F}$ é proporcional à redução da CVF, e o Índice de Tiffeneau (VEF1/CVF) é normal ou mesmo aumentado ${ }^{16}$

Testes de função pulmonar regulares são necessários para monitorar a progressão da falência respiratória. A American Academy of Neurology recomenda medir a CVF no dia do diagnóstico e a cada três meses ${ }^{9,25}$.

As vantagens são de um instrumento viável, econômico e de fácil realização com um simples equipamento portátil, mas depende da colaboração do paciente para a sua adequada realização ${ }^{18}$. Exceto pela variação en- 
tre posição sentada e supina, a espirometria é pouco sensível e específica. A sensibilidade é baixa porque as alterações só aparecem quando a força muscular é menor que um terço do normal. A especificidade é baixa, pois várias outras doenças pulmonares causam alterações espirométricas mesmo com a função muscular normal (fibrose, DPOC, obesidade). Além disso, uma baixa CVF em pacientes com comprometimento bulbar pode ser subestimada e não significar a real fraqueza, por isso mais testes podem ser necessários ${ }^{11}$.

\section{Curvas fluxo-volume}

Alterações da curva fluxo-volume podem ser encontradas nas DNM, como queda abrupta no final da curva expiratória, indicando intensa fraqueza dos músculos expiratórios ${ }^{15}$; rompimento abrupto do pico de fluxo inspiratório ou expiratório e a lentidão para atingir o pico de fluxo expiratório possuem $90 \%$ de sensibilidade e $80 \%$ de especificidade em predizer fraqueza respiratória26; e oscilação nas curvas possuem 90\% de especificidade e $80 \%$ de sensibilidade em predizer fraqueza dos músculos das vias aéreas superiores ${ }^{26,27}$.

\section{Ventilação voluntária máxima (VVM)}

A VVM é um teste de esforço, realizado durante a espirometria, que representa os fluxos inspiratório e expiratório máximos. É determinada pela condutância das vias aéreas, força muscular e volume mobilizado a cada respiração ${ }^{14}$, e é um dos primeiros indicadores de fraqueza muscular respiratória ${ }^{10}$, diretamente relacionada com a CVF ${ }^{28}$, mas as medidas das pressões respiratórias não podem ser substituídas pela determinação da WVM, pois a VVM pode estar alterada apenas quando a força muscular média estiver menor que 37\% do normal ${ }^{16}$.

O paciente deve respirar o maior volume corrente possível durante 12 a 15 segundos na faixa intermediária da CVF, fator importante para a fase expiratória pois, a retração elástica pulmonar pode estar levemente aumentada. Os valores alcançados por indivíduos normais variam de 50 a $250 \mathrm{l} / \mathrm{min}$, porém o limite inferior para descartar disfunção ventilatória é de $10 \mathrm{l} / \mathrm{min}^{15}$.

A avaliação da resistência é mais importante que a da força muscular respiratória, na verdade medimos a força dos músculos respiratórios como uma medida indireta da resistência. E por que não medir a resistência? Porque para manter a ventilação em isocapnia são necessários equipamentos sofisticados que ofereçam estímulo visual aos pacientes ${ }^{15}$. E, é importante lembrar que em se tratando de pacientes com DNM, o limiar de fadiga esta reduzido ${ }^{16}$.

\section{Força Muscular Respiratória (FMR)}

Na presença de DNM, a FMR pode estar reduzida mesmo com os volumes pulmonares normais e, o oposto ocorre na presença de deformidades torácicas, quando a FMR está normal com volumes e capacidades reduzidos ${ }^{29}$. Para determinar a causa primária desta disfunção, a análise das propriedades dos músculos respiratórios deve ser realizada associada as alterações de volumes e capacidade pulmonares.

As variações de pressões na caixa torácica e na cavidade abdominal representam a contração dos músculos respiratórios. Estas pressões podem ser avaliadas pelas vias aéreas superiores (boca, nariz e faringe), esôfago e estômago durante manobras com contrações voluntárias ou involuntárias.

Nos pacientes com DNM, ao realizar muitos testes em um mesmo período, deve-se considerar a predisposição à fadiga ${ }^{30}$.

\section{Pressão inspiratória e expiratória máximas}

O teste mais utilizado para avaliar a força muscular inspiratória e expiratória são as medidas de pressão inspiratória e expiratória máximas estática de boca (PImáx e PEmáx) $)^{5,13}$

A medida da PImáx pode ser realizada a partir da CRF ou VR e a PEmáx é, convencionalmente medida a partir da capacidade pulmonar total ${ }^{31}$.

O valor da PImáx representa a pressão desenvolvida pelos músculos respiratórios mais a pressão de recolhimento elástico da parede torácica e do tecido pulmonar. Quando medida a partir do volume residual, existe uma desvantagem teórica causada pelo recolhimento elástico da parede torácica que contribui para os valores obtidos ${ }^{15}$ mas, na prática, os pacientes referem que a manobra é mais fácil de ser realizada a partir do volume residual em comparação com a capacidade residual funcional ${ }^{13}$.

Um manovacuômetro ou transdutor de pressão portátil permite medir a PImáx e PEmáx, tanto em ambulatório como na beira do leito, com a vantagem de ser um teste não invasivo ${ }^{15}$. Estas medidas são realizadas na posição sentada utilizando um clipe nasal e um bucal padrão com um orifício de $2 \mathrm{~mm}$ para evitar artefatos de pressão causados pelos músculos faciais ${ }^{30}$.

O paciente inspira e expira com o máximo de força contra um circuito ocluído conectado ao manovacuômetro, manobra de Muller e Valsava, respectivamente. Os maiores valores de pressão obtidos durante 1 segundo representam os valores de PImáx e PEmáx a serem considerados ${ }^{15}$. Em qualquer um dos métodos são feitas 3 medidas, considerando-se o maior valor obtido daqueles que apresentarem variação menor que $20 \%{ }^{13}$.

Os valores de normalidade para PImáx esta em torno de - $80 \mathrm{cmH}_{2} \mathrm{O}$ para homens e - $60 \mathrm{cmH}_{2} \mathrm{O}$ para mulheres e, valores de PEmáx de $150 \mathrm{cmH}_{2} \mathrm{O}$ para homens e $120 \mathrm{cmH}_{2} \mathrm{O}$ para mulheres. Uma PImáx com uma alta negatividade de $-60 \mathrm{cmH}_{2} \mathrm{O}$ ou um valor de PEmáx com 
uma alta positividade de $+90 \mathrm{cmH}_{2} \mathrm{O}$, de testes tecnicamente satisfatórios excluem fraqueza dos músculos respiratórios, se associada à medida de CVF32.

A principal vantagem deste teste volitivo é o de estimar a força muscular de maneira simples e bem tolerada pelos pacientes, apesar de não garantir que esta sendo realizado um esforço realmente máximo. Além disso, a PImáx e PEmáx são influenciadas pela idade, sexo, postura, volume pulmonar e o tipo de bucal' ${ }^{32}$.

Durante o progredir da fraqueza dos músculos respiratórios, a perda de força muscular ocorre antes da redução do volume pulmonar ser detectada, sendo assim, a PImáx pode ser mais sensível que a CVF ${ }^{31}$.

Fatores como esforço submáximo ou vazamento de ar ao redor do bucal podem gerar medidas errôneas, principalmente em indivíduos com fraqueza nos músculos orofaciais. Por este motivo, manobras simplificadas ou medidas que não requerem uso da musculatura orofacial têm sido propostas como meios alternativos para medir a FMR, assim como, o teste de inspiração nasal máxima, um método mais fácil de realizar em comparação com a PImáx, o que permite valores mais fidedignos.

\section{Pressão nasal máxima (Sniff Nasal Pressure - Pnas,sn)}

A medida da pressão nasal após uma inspiração nasal máxima, conhecida como "sniff nasal pressure"(Pnas,sn), baseia-se na transmissão de pressão homogênea por todo espaço pleural, esôfago, nasofaringe e nariz ${ }^{29}$. Durante esta manobra ocorre um pequeno aumento do volume corrente de $500 \mathrm{ml}$ e, o diafragma possui uma contração relativamente isométrica ${ }^{13}$.

A medida é feita com um cateter conectado a um transdutor de pressão e introduzido em uma das narinas, com a narina oposta obstruída e a boca fechada ${ }^{15}$.

O paciente deve ser orientado a permanecer sentado e realizar uma inspiração nasal máxima e forte (cheirar) a partir da CRF, 10 vezes e com repouso de 30 segundos, considerando o maior valor de pressão obtido ${ }^{29}$.

As medidas também podem ser feitas com um cateter esofágico (Pes,sn), realizando a mesma manobra, porém com a desvantagem de um método invasivo, ${ }^{9,13}$. A Pes,sn representa a pressão pleural e não tem interferência do recolhimento elástico do pulmão, refletindo a pressão gerada pelos músculos inspiratórios ${ }^{15}$.

Valores da Pnas, sn mais negativos que $-70 \mathrm{cmH}_{2} \mathrm{O}$ para homens e $-60 \mathrm{cmH}_{2} \mathrm{O}$ para as mulheres excluem fraqueza dos músculos inspiratórios ${ }^{29}$.

Os valores de normalidade da Pes,sn durante uma inspiração nasal máxima são mais negativos que
- $80 \mathrm{cmH}_{2} \mathrm{O}$ para os homens e - $70 \mathrm{cmH}_{2} \mathrm{O}$ para as mulheres ${ }^{13}$, com uma diferença de $10 \mathrm{cmH}_{2} \mathrm{O}$ entre as medidas nasal e o esofágico.

A pressão transdiafragmática também pode ser avaliada durante uma inspiração nasal máxima (Pdi,sn), representando a força muscular do diafragma. Essa manobra necessita de um cateter duplo com balão esofágico e gástrico. $\mathrm{O}$ valor de normalidade é maior do que $100 \mathrm{cmH}_{2} \mathrm{O}$ para homens e maior do que 80 $\mathrm{cmH}_{2} \mathrm{O}$ para mulheres ${ }^{15}$.

A Pnas,sn possui a vantagem de ser uma manobra natural, não invasiva e de fácil realização ${ }^{29,13}$. Em adultos, foi observada uma maior ativação do diafragma durante uma inspiração nasal máxima em comparação com a manobra de Plmáx, o que torna a manobra mais fidedigna e reprodutíve $e^{130,33}$.

A Pes,sn é uma avaliação da FMR global, enquanto que a Pdi,sn é a medida específica da força diafragmática ${ }^{13}$. Estas são adequadas para pacientes com comprometimento bulbar ${ }^{9}$.

Pressões: esofágica, gástrica, transdiafragmática e índice de atividade diafragmática.

Medidas não voláteis da FMR podem ser feitas através de métodos invasivos, analisando-se as pressões torácicas e abdominais ${ }^{34}$, mensurando as pressões esofágica (Pes), gástrica (Pga), transdiafragmatica $(\mathrm{Pdi})^{13}$. A Pes reflete a pressão pleural, a Pga reflete a pressão abdominal e a Pdi representa a força diafragmática ${ }^{15}$.

Para a análise da Pes e Pga é necessário inserir um cateter duplo de polietileno, com dois balões na extremidade distal de cada cateter, um no terço médio do esôfago e outro no interior do estômago, ambos conectados a transdutores de pressão ${ }^{34}$. O volume dos balonetes, características de pressão-volume e dimensão do cateter podem influenciar as medidas das pressões e causar erros ${ }^{15}$.

Os traçados das Pes e Pga diferem muito entre indivíduos normais, mas quando analisados em pacientes com DNM, estes traçados são muito similares ${ }^{31}$.

A Pdi é a diferença entre Pga (pressão abdominal) e Pes (pressão pleura): Pdi = Pga - Peso e é considerada o Gold Standart da medida da força muscular diafragmática ${ }^{34}$.

Durante uma inspiração até CPT, a Pdi pode alcançar no mínimo $40 \mathrm{cmH}_{2} \mathrm{O}$ em indivíduo normal ${ }^{34} \mathrm{e}$, pode variar até $10 \mathrm{cmH}_{2} \mathrm{O}$ quando o paciente passa de sentado para a posição supina ${ }^{30}$.

A atividade motora do diafragma pode ser analisada com o índice de Gilbert, variação da pressão gástrica sobre a variação da Pdi durante uma inspiração completa $(\triangle \mathrm{Pga} / \triangle \mathrm{Pdi})$. Este índice normalmente é positivo, mas se torna negativo caso a pressão abdominal cair durante 
a inspiração, sugerindo uma respiração paradoxal. Uma média dos valores, adquiridos durante 2 minutos de respiração tranqüila pode ser utilizada na análise dos dados $^{35}$. Determina a presença de respiração paradoxal, atividade motora diafragmática e possui maior relação com os valores espirométricos do que com a Plmáx ou PEmáx ${ }^{30}$.

A vantagem destes testes é a não dependência de atividade voluntária em comparação com a Pdi, PImáx e Pnas,sn. As desvantagens são a interferência da atividade abdominal e, ser um teste invasivo ${ }^{6}$

\section{Estimulação Elétrica Magnética (ou eletromagné- tica?) do Nervo Frênico (EEM)}

Permite estudar as duas hemicúpulas diafragmáticas ou cada uma isoladamente, excluindo os outros músculos respiratórios ${ }^{15}$.

É necessário localizar no pescoço o local por onde passa o nervo frênico, para o posicionamento do eletrodo, enviando estímulos elétricos dosados e reprodutíveis. O estímulo é aumentado gradativamente até que seja atingido um valor supramáximo, ou seja, a partir deste ponto, mesmo aumentando a descarga elétrica, não há aumento da força gerada. A força gerada pode ser obtida através de Pes, Pdi ou da pressão em via aérea proximal (boca, cateter nasal ou tubo traqueal)36.

A série normal descrita é extensa para a Pdi durante a EEM do nervo frênico $\left(8.8-33 \mathrm{cmH}_{2} \mathrm{O}\right)$ sem diferença entre o sexo masculino e feminino. Um diagnóstico útil para fraqueza muscular grave de pacientes incapazes de realizar testes voláteis e volitivos ${ }^{15}$.

Este é um método difícil de realizar, pois se o estímulo elétrico não for exatamente no mesmo local, há muita variação na pressão gerada e, em pacientes acordados o estímulo elétrico pode ser doloroso ${ }^{13}$.

\section{Estimulação Magnética do Nervo Frênico (EM)}

A EM é realizada da mesma forma que a EEM, mas não é dolorosa e não depende do posicionamento dos eletrodos tanto como na EEM. Por outro lado, não estimula só o diafragma, mas também outros músculos respiratórios ${ }^{36}$.

A estimulação supramáxima do nervo frênico, bilateralmente pode ser atingida utilizando-se uma bobina circular colocada acima da raiz cervical do nervo frênico. A descarga da bobina gera um campo de pulso magnético, que causa uma corrente no tecido nervoso, sem que o campo ao redor cause contração muscular. E a estimulação bilateral, quando anterior, permite o estudo da função diafragmática na posição supina $^{15,36}$.

A Pdi durante a EM do nervo frênico atinge valores de $20 \mathrm{cmH}_{2} \mathrm{O}$, sem diferença entre o sexo masculino e feminino ${ }^{15}$.

Este teste possui limite inferior de normalidade mais bem definido que a EEM, além de uma maior relação com a Pdi, o que aumenta a sensibilidade diagnóstica em pacientes com fraqueza muscular moderada ${ }^{13}$.

\section{Eletromiografia (EMG) dos Músculos Respira- tórios}

A EMG é o registro das ondas elétricas geradas pelo músculo estudado e dos nervos que o estimulam, portanto, serve para avaliar aspectos da inervação, junção neuromuscular e atividade intrínseca dos músculos. Permite estudar separadamente o diafragma e os músculos torácicos, além de diferenciar as formas de fadiga e avaliar aquelas de maior importância para os músculos respiratórios, como a fadiga aos estímulos de baixa freqüência $(2 \mathrm{OHz})^{15}$.

Antes de qualquer alteração clínica, o teste de EMG já mostra o aparecimento de fadiga, que se caracteriza pela troca de sinais elétricos de alta freqüência por de baixa freqüência ${ }^{37}$.

A vantagem da EMG esta em não ser um método invasivo e, não depender da colaboração do paciente. A desvantagem é que se trata de um exame difícil de se realizar e pouco acessível ${ }^{37}$.

\section{Fadiga Muscular Respiratória}

A fadiga muscular respiratória pode causar falência da bomba ventilatória, se a demanda metabólica exceder o suprimento energético. A máxima força de um músculo e a carga a ele imposta são importantes determinantes de fadiga e resistência musculares.

A fadiga ocorre mais freqüentemente, quando a pressão muscular máxima esta reduzida ou quando a pressão necessária em qualquer respiração está aumentada, como quando ocorre aumento da resistência e redução da complacência².

A pressão transdiafragmática para realizar um dado volume corrente (Pdi) sobre a pressão transdiafragmática em uma inspiração máxima (Pi,di,max), permite avaliar a pressão necessária para manter uma ventilação adequada. O tempo que esta pressão é gerada pode ser expresso pela relação entre o tempo inspiratório (Ti) e o tempo total do ciclo respiratório (Ttot). Destas duas relações temos o índice Tensão-Tempo (TTdi), que é definido pela equação: TTdi $=$ Pdi/Pi,di,máx $\times$ Ti/Ttot. O limiar de fadiga muscular respiratória em indivíduos normais ocorre quando TTdi é maior que 0.15 ou quando Pdi/Pdimáx é igual ou maior que 0.438

\section{Relaxamento muscular do diafragma}

Músculos fadigados relaxam lentamente. O relaxamento do diafragma pode ser analisado a partir da 
redução na Pdi. A demora no grau de relaxamento dos músculos respiratórios, durante uma inspiração nasal máxima analisa a recuperação muscular. O relaxamento do diafragma, após uma inspiração nasal máxima, também pode ser analisado a partir dos traçados da Pes, da pressão na nasofaringe ou na boca ${ }^{39}$.

\section{Avaliação da eficácia tosse: Pico de Fluxo Expi- ratório e Pico de Fluxo de Tosse}

Uma tosse eficaz requer expiração forçada contra a glôte fechada, causando um fluxo expiratório explosivo após a abertura da glôte. Portanto, depende de força muscular inspiratória, expiratória e da integridade dos músculos de inervação bulbar ${ }^{40}$.

Ao contrário dos inúmeros testes de força muscular inspiratória, poucos testes foram desenvolvidos para acessar a força muscular expiratória. Apenas, o teste de PEmáx possui valores de normalidade estabelecidos, mas com limitações similares à Pimáx. Para contornar essas limitações, os valores de Pga e Pes durante uma tosse máxima têm sido estudados, de maneira a adquirir medidas não voláteis da força muscular expiratória ${ }^{13}$. Foram encontrados valores de Pga maiores que 160 $\mathrm{cmH}_{2} \mathrm{O}$ para os homens e maiores que $120 \mathrm{cmH}_{2} \mathrm{O}$ para as mulheres, sendo a Pes numericamente similar a Pga durante a tosse ${ }^{41}$.

A fraqueza dos músculos expiratórios também pode causar redução do fluxo expiratório e do fluxo de tosse. O pico de fluxo expiratório (PFE) e de tosse (PFT), na ausência de obstrução brônquica possuem um comportamento dinâmico com relação direta ao comportamento estático da PEmáx, com $\mathrm{p}<0.00140$.

Os valores de PFE e PFT são obtidos com um medidor de fluxo (pneumotacógrafo). As medidas devem ser realizadas com o paciente sentado, utilizando um clipe nasal e orientado a tossir o mais forte possível, a partir da CPT. Devem ser realizadas três manobras, considerando o maior valor obtido. O valor de referência do PFT é maior que $160 \mathrm{l} / \mathrm{min}$ e para o PFE maior que $100 \mathrm{l} / \mathrm{min}$, para ambos os sexos ${ }^{40}$.

A desvantagem destes testes em pacientes com DNM, esta em refletir uma pobre coordenação dos músculos respiratórios ao invés de perda de força muscular respiratória per $\mathrm{se}^{15}$.

\section{Gasometria arterial}

No início do comprometimento respiratório em pacientes com DNM, a alteração dos gases acontece durante o sono, por conta da hipotonia fisiológica durante esta fase. Quando há hipercapnia causada exclusivamente por fraqueza muscular, esta deve ser muito grave e o prognóstico do paciente costuma ser ruim ${ }^{42}$.

A pressão arterial de dióxido de carbono ( $\mathrm{PaCO} 2)$ é considerada alta quando $>45 \mathrm{mmHg}$, a pressão arterial de oxigênio (PaO2) é considerada baixa quando <
80mmHg e a saturação arterial de oxigênio (SatO2) é considerada reduzida quando menor que 90\%43. Durante a fraqueza moderada dos músculos respiratórios pode ocorrer pequena hipercapnia, mas quando a força muscular esta reduzida em $40 \%$ do predito o aumento do PaCO2 é maior ${ }^{42}$.

A gasometria arterial em pacientes com DNM é um teste pouco sensível, porque somente apresenta alteração quando a FMR é menor que $30 \%$ do normal, e pouco específico por estar alterado em outras doenças pulmonares ${ }^{44}$.

\section{Capnografia}

Capnografia é a análise gráfica da concentração de CO2 sobre o tempo. Em geral, essa análise ocorre a cada ciclo respiratório. Atualmente, pode ser realizada por duas técnicas: espectrometria de massa e absorção de luz infravermelha ${ }^{45}$.

Em um indivíduo normal, a pressão parcial de dióxido de carbono ao final da expiração (PetCO2) mantém relação constante com $\mathrm{PaCO} 2$, sendo a diferença entre PaCO2 e PetCO2 menor que $5 \mathrm{mmHg}$, sendo a diferença maior que $10 \mathrm{mmHg}$ indicativo de comprometimento pulmonar ${ }^{45}$.

A elevação da PaCO2 implica grave fraqueza muscular respiratória, particularmente do diafragma, com pobre prognóstico ${ }^{2}$. Pacientes com hipercapnia sintomática noturna são, também grandes candidatos para o suporte ventilatório, mesmo na ausência de hipercapnia durante o dia ${ }^{46}$.

\section{Oximetria de pulso}

O oxímetro de pulso baseia-se na espectrofotometria de acordo com o comprimento de onda de luz (600 a 1000 nm), com um sensor que possui um pólo de luz e outro de registro da onda. Este sensor mede a saturação de pulso da hemoglobina (SapO2) ${ }^{42}$.

Pacientes com DNM evoluem com hipoventilação, o que primariamente aumenta a retenção de $\mathrm{CO}_{2}$, mas sem apresentar hipoxemia. A hipoxemia pode ocorrer quando a disfunção ventilatória atinge estágios mais avançados, ou na presença de doenças associadas como a pneumonia ${ }^{7}$

A oximetria noturna permite identificar alterações significativas para o diagnóstico de hipoventilação, quando a $\mathrm{SatO}_{2}$ cai abaixo de $80 \%$, principalmente durante o sono REM ${ }^{46}$.

\section{CONCLUSÃO}

Para a avaliação da função pulmonar em pacientes com doenças neuromusculares, foram encontrados nove tipos de testes, tais como: da função pulmonar, 
que inclui medidas espirométricas (CVF, VEF $F_{1}, \mathrm{CVFNEF}_{1}$ FEF 25-75\%, análise da curva fluxo-volume e VVM) utilizados para avaliar volumes pulmonares, presença de obstrução brônquica, sinais de fraqueza e fadiga dos músculos respiratórios; de força dos músculos respiratórios (PImáx, PEmáx, Pnas,sn, Pes,sn e Pdi,sn, Pes, Pga e Pdi, $\triangle \mathrm{Pga} / \triangle \mathrm{Pdi}$, Pdi durante EEM e EM do nervo frênico), utilizados para avaliar a força dos músculos respiratórios e a atividade diafragmática; da atividade elétrica de nervos, junção neuromuscular e músculos
(EMG dos músculos respiratórios); do limiar de fadiga dos músculos respiratórios (TTdi e Pdi/Pdimáx); do grau de relaxamento dos músculos respiratórios (diminuição da Pdi), utilizado para analisar a recuperação muscular; da eficácia da tosse (PFT e PFE); análise da concentração de gases arterias (PaCO2, $\mathrm{PaO} 2$, SatO2), capnografia (PetCO2 - PaCO2), utilizada para avaliar, principalmente a retenção de $\mathrm{CO}_{2}$ e; oximetria de pulso ( SapO2), utilizada para avaliar presença de hipoxemia, principalmente durante o sono.

\section{REFERÊNCIAS BIBLIOGRÁFICAS}

1. Calia LC, Annes M. Afecções neurológicas periféricas. Levy JA, Oliveira ASB. Reabilitação em Doenças Neurológicas. São Paulo: Atheneu; 2003, p31-64.

2. Syabbalo N. Respiratory Muscle Function in patients with neuromuscular disorders and cardiopulmonary diseases. Int J Clin Pract 1998; 52: 319-329.

3. Stambler N, Charatan M, Cedarbaum JM. Prognostic indicators of survival in ALS. Neurology 1998; 50: 66-72.

4. Gozal D. Pulmonary manifestations of neuromuscular disease with special reference to duchenne muscular dystrophy and spinal muscular atrophy. Pediatr Pulmonol 2000; 29: 141-150.

5. Perrin C, Unterborn JN, Ambrosio C, Hill NS. Pulmonary complications of chronic neuromuscular disease and their management. Muscle Nerve 2004; 29: 5-27.

6. Rochester DF, Sharon AE. Assessment of ventilatory function in patients with neuromuscular disease. Clin Chest Med 1994; 15: 751-763.

7. Poponick JM, Jacobs I, Supinski G, Dimarco AF. Effect of upper respiratory tract infection in patients with neuromuscular disease. Am J Respir Crit Care Med 1997; 156: 659-664

8. Lyall RA, Donaldson N, Polkey MI, Leigh PN, Moxham J. Respiratory muscle strength and ventilatory failure in amyotrophic lateral sclerosis. Brain 2001 124: 2000-2013

9. Lechtzin N, Rothstein J, Clawson L, Diette GB, Wiener CM. Amyotrophic lateral sclerosis: evaluation and treatment of respiratory impairment ALS and othe motor neuron disorders 2002; 3: 5-13

10. Kaplan LM, Hollander D. Respiratory dysfunctions in amyotrophic latera sclerosis Clin Chest Med 1994; 15: 675-681.

11. Hopkins LC, Tatarian GT, Pianta TF. Management of ALS: Respiratory care Neurology 1996; 47: S123-S125.

12. Similowski T, Attali V, Bensimon G. Diaphragmatic dysfunction and dyspneia in amyotrophic lateral sclerosis. Eur Respir J 2000; 15: 332-337.

13. Polkey MI, Green M, Moxham J. Measurement of respiratory muscle strength Thorax 1995; 50: 1131-1135.

14. American Association for Respiratory Care Clinical. Pratice Guideline: Spirometry. Respir Crit Care Med 1996; 41: 629-636.

15. American Thoracic Society/ European Respiratory Society - ATS/ERS. Statement on respiratory muscle testing. Am J Respir Crit Care Med 2002; 166 518-624.

16. Mendonça EMC, Pereira CAC. Mecânica pulmonar nas doenças neuromusculares. J Pneumol. 1984; 10: 223-232.

17. Kristufek P, Brezina M, Ciutti P, Strmen J, Mayer M. Reference values and modeling of lung function development as a transcendent function of age, body, height and mass. Bull Eur Phys Respir 1987; 23: 139-147.

18. Pereira CAC, Barreto SP, Simões JG, Pereira FWL, Gerstler JG, Nakatani J. Valores de referência para a espirometria em uma amostra da população brasileira adulta. J Pneumol 1992; 18: 10-22

19. Costa FS, Chueiri EB, Silva WCS, Pereira C, Nakatani J. Valores de referência para espirometria em uma amostra da população brasileira adulta da raça negra. J Pneumol 1996; 22: 165-170.

20. Baur X, Isringhausen S, Degens P. Comparison of lung-function reference values. Int Arch Occup Environ Health 1999; 72: 69-83.

21. Quanjer AD, Zomeren BC. Summary equation of reference values. Bull Europ Phys Resp 1983; 19: 45-51.

22. Subbarao P, Lebecque P, Corey M, Coates AL. Comparison of spirometric reference values. Pediatr Pulmonol 2004; 37: 515-522.

23. Dockery DW, Ware JH, Ferris Jr BG, Glicksberg DS, Fay ME, Spiro III A, et al. Distribution of forced spirometric volumes in one second and forced vital capacity in health, white, adult never smokers in six U.S. cities. Am Rev Respir Dis 1985; 131: 511-520.

24. Quanjer PH, Borsboom GJ, Brunekreff B, Zach M, Forche G, Cotes JE, et al. Spirometric reference values for white european children and adolescents. Pediatr Pulmonol. 1995; 19: 135-42.
25. Miller RG, Rosemberg JA, Gelinas DF. Pratice parameter: the care of pacients with ALS (a evidence based review). Report of the quality standards subcommittee of American Academy of Neurology. Neurology 1995; 52 1311-1323.

26. Vincken WG, Elleker MG, Cosio MG. Flow-volume loop changes reflecting respiratory muscle weakness in clinical neuromuscular disorders. Am J Med 1987: 83: 673-680.

27. Vincken WG, Ellerker MG, Cosio MG. Detection of upper airway muscle involvement in neuromuscular disorders using the flow-volume curve. Chest 1986; 90: 52-57.

28. Gelinas DF. Vital capacity as an efficacy measure: Summary.

Amyotroph Lateral Scler Other Motor Neuron Disord 2002; 3: S61-S62.

29. Stefanutti D, Fitting JW. Sniff nasal inspiratory pressure. Am J Respir Crit Care Med 1999; 159: 107-111

30. Fromageot C, Lofaso F, Annane D, Falaize L, Lejaille M, Clair B, et al. Supine fall in lung volumes in the assessment of diaphragmatic weakness in neuromuscular disorders. Arch Phys Med Rehabil 2001; 82: 123-128.

31. Lyall RA, Donaldson N, Polkey MI, Leigh PN, Moxham J. Respiratory muscle strength and ventilatory failure in amyotrophic lateral sclerosis. Brain 2001 124: 2000-2013.

32. Bruschi C, Cervai I, Zoia MC, Fanfulla F, Fiorentini M, Cosali L, et al. Reference values of maximal inspiratory mouth pressure: a population based study. Am Rev Respir Dis 1992; 146: 790-793.

33. Nara S, Ambrosino N, Crotti D, Franchia C, Dampella C. Recruitment of some respiratory muscles during the maximal inspiratory maneuver. Thorax 1993 48: $702-707$

34. Laporta D, Grassino A. Assessment of transdiaphragmatic pressure in humans J Appl Physiol 1985; 58: 1469-1476.

35. Gilbert R, Auchinchors J, Reppi P. Relationship of rib cage and abdomen motion to diaphragm function during quite breathing. Chest 1995; 80: 607-612.

36. Man WDC, Moxham J, Polkey MI. Magnetic stimulation for the measurement of respiratory and skeletal muscle function. Eur Respir J 2004; 24: 846-860.

37. Stewart H, Eisen A, Road J, Mezei M, Weber M. Electromyography of respiratory muscle in amyotrophic lateral sclerosis. J Neurol Sci 2001; 191: 67-73.

38. Bellemere F, Grassino A. Effect of pressure and timing of contraction on human diaphragmatic fatigue. J Appl Physiol 1995; 53: 1190-1195.

39. Koulouris N, Vianna LG, Mulvey DA. Maximal relaxation rates of esophageal, nose and mouth pressures during sniff reflect inspiratory muscle fatigue. Am Rev Resp Dis 1985; 139: 1213-1217.

40. Suárez AA, Pessolano FA, Monteiro SG, Ferreyra G, Capria ME, Mesa L, et al. Peak Flow and Peak Cough Flow in the evaluation of expiratory muscle weakness and bulbar impairment in patients with neuromuscular disease. Am J Phys Med Rehabil 2002; 81: 506-511.

41. Polkey MI, Lyall RA, Green M, Leigh N, Moxham J. Expiratory muscle function in amyotrophic lateral sclerosis. Am J Respir Crit Care Med 1998; 158 734-741.

42. Hecht MJ, Brow CM, Mittelhamm F, Werner D, Heuss D, Neundorfer B, et al Increased hypoxic blood pressure response en patients with amyotrophic lateral sclerosis. J Neurol Sci 2003; 213: 47-53.

43. Misuri G, Lanini B, Gigliotti F, landelli I, Pizzi A, Bertlini MG, et al. Mechanism of $\mathrm{CO} 2$ retention in patients with neuromuscular disease. Chest 2000; 117 447-553.

44. Ragette R, Mellies U, Schwake C, Voit T, Teschler H. Patterns and predictors of sleep disordered breathing in primary myopathies. Thorax 2000; 57 724-728

45. Kotterba S, Patzold T, Malin JP, Orth M, Rasche K. Respiratory monitoring in neuromuscular disease - capnography as an additional tool? Clinical Neurosurgery 2001; 103: 87-91.

46. Hukins CA, Hillman DR. Daytime predictors of sleep hypoventilation in duchenne muscular dystrophy. Am J Respir Crit Care Med 2000; 161: 166-170. 